\title{
Evolution of the ROOT Tree I/O
}

\author{
Jakob Blomer ${ }^{1, *}$, Philippe Canal $^{2}$, Axel Naumann ${ }^{1}$, and Danilo Piparo ${ }^{1}$ \\ ${ }^{1}$ CERN, Geneva, Switzerland \\ ${ }^{2}$ Fermilab, Chicago, U.S.
}

\begin{abstract}
The ROOT TTree data format encodes hundreds of petabytes of High Energy and Nuclear Physics events. Its columnar layout drives rapid analyses, as only those parts ("branches") that are really used in a given analysis need to be read from storage. Its unique feature is the seamless $\mathrm{C}++$ integration, which allows users to directly store their event classes without explicitly defining data schemas. In this contribution, we present the status and plans of the future ROOT 7 event I/O. Along with the ROOT 7 interface modernization, we aim for robust, where possible compile-time safe $\mathrm{C}++$ interfaces to read and write event data. On the performance side, we show first benchmarks using ROOT's new experimental I/O subsystem that combines the best of TTrees with recent advances in columnar data formats. A core ingredient is a strong separation of the high-level logical data layout $(\mathrm{C}++$ classes) from the low-level physical data layout (storage backed nested vectors of simple types). We show how the new, optimized physical data layout speeds up serialization and deserialization and facilitates parallel, vectorized and bulk operations. This lets ROOT I/O run optimally on the upcoming ultra-fast NVRAM storage devices, as well as file-less storage systems such as object stores.
\end{abstract}

\section{Introduction}

The data describing a High Energy Physics (HEP) event is typically represented by a record containing variable-length collections of sub records. An event can, for instance, contain a collection of particles with certain scalar properties ( $p_{t}, E$, etc.), another collection of jets, a collection of tracks, and so on. A typical physics analysis uses a large number of events but processes only a subset of the available properties. Therefore, ROOT's TTree storage format support a columnar physical data layout for nested sub records and collections [1]. Values of a single property of many events (e.g., $p_{t}$ for events 1 to 1000) are stored consecutively on disk. Thus, only those parts that are required for an analysis need to be read. Similar values are likely to be grouped together, which is beneficial for compression.

More than $1 \mathrm{~EB}$ of data is stored in the TTree format. For HEP use cases, the TTree I/O speed and storage efficiency has shown to be significantly better than many industry products [2]. Furthermore, ROOT provides the unique feature of seamless $\mathrm{C}++$ and Python integration where users do not need to write or generate a data schema. Yet, the TTree implementation limits the optimal use of new storage systems and storage device classes, such as object stores and flash memory, and it shows shortcomings when it comes to multithreaded and GPU supported analysis tasks and fail-safe APIs.

\footnotetext{
*e-mail: jblomer@cern.ch
} 
In this contribution, we present the design and first benchmarks of the RNTuple set of classes. The RNTuple classes provide a new, experimental columnar event I/O system that is backwards-incompatible to TTree both on the file format level and on the API level. Breaking backwards compatibility allows us to use contemporary nomenclature and to design the ROOT event $\mathrm{I} / \mathrm{O}$ from the ground up for next-generation devices and the increased data rates expected from HL-LHC. Once the new I/O sub system has matured, we expect new experiment data to be written in RNTuple format while we intend to keep the TTree I/O system operational for processing existing data sets.

\section{Design of the RNTuple I/O subsysem}

This section describes key design choices of the RNTuple data format and of class design and the interfaces.

\subsection{Data layout}

Compared to the TTree binary data layout, the RNTuple data layout is modestly modernized and borrows some ideas from Apache Arrow [3] (see Figure 1). Data is stored in columns of fundamental types supporting arbitrarily deeply nested collections (TTree drops the "columnar-ness" for deeply nested collections). ${ }^{1}$ Columns are partitioned in compressed pages, of typically a few tens of kilobytes in size. Like in TTree, clusters are a set of pages that contain all the data of a certain event range. They are typically a few tens of megabytes in size and a natural unit of processing for a single thread or task.

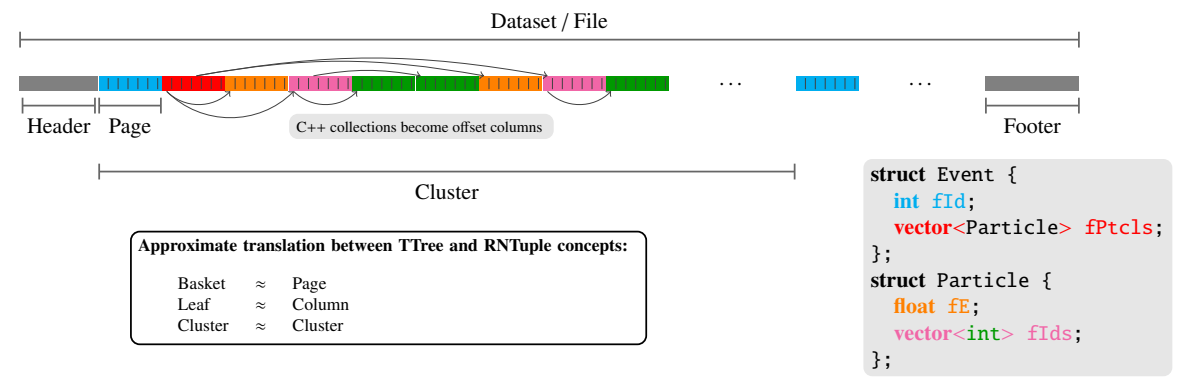

Figure 1. Breakdown of the RNTuple data layout. Each scalar field of the event struct is stored in a separate column.

A collection's representation contains an offset column whose elements indicate the start index within the columns that store the collection content; this allows for random-access of individual events. The indexing is local to the cluster such that clusters can be written in parallel and freely concatenated to a larger data set. This also allows for "fast merging", where several RNTuple files can be concatenated by only adjusting the header and footer. In contrast to TTree, offset pages and value pages are always separated, which should improve the compression ratio (to be confirmed). Integers and floating point numbers in columns are stored in little-endian format (TTree: big-endian) in order to allow for memory mapping of pages on most contemporary architectures, in partiular x86_64 as well as AArch64 and POWER in little-endian mode. Boolean values, such as trigger bits, are stored as bitmaps (TTree: byte arrays), which improves the compression.

\footnotetext{
${ }^{1}$ In contrast to TTree, RNTuple currently does not support row-wise storage.
} 
The RNTuple meta-data are stored in a header and a footer. The header contains the schema of the RNTuple; the footer contains the locations of the pages. At a later point, we will extend the meta-data with a regularly written checkpoint footer (e.g. every $100 \mathrm{MB}$ ) in order to allow for data recovery in case of an application crash during data taking. We will also extend the meta-data with a user-accessible, namespace-scoped map of key-value pairs, such that the experiment data management systems can maintain relevant information (checksums, replica locations, etc.) together with the data.

The pages, header and footer do not necessarily need to be written consecutively in a single file. As the footer contains the page locations, an RNTuple can be stored using different container formats as long as the container specifies the location (entry point) of the header and footer. In the common case of the container being a ROOT file, dedicated TKeys are used for the header and footer. It is also conceivable to store header and footer in a different file than the pages to avoid backward seeks. A similar approach can be used to store an RNTuple in an object store: a well-known key provides header and footer, which in turn contain the object addresses of the pages.

\subsection{Class design}

The RNTuple class design comprises four layers (see Figure 2). The RNTuple classes make use of templates, such that for simple types (e.g., vectors of floats) that are known at compile time, the compiler can inline a fast path from the highest to the lowest layer without additional value copies or virtual calls.

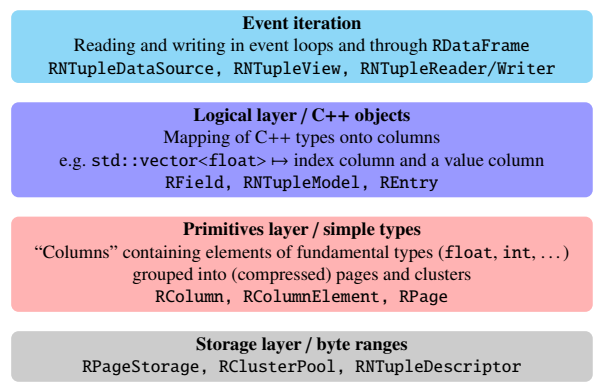

\begin{tabular}{|c|c|c|}
\hline \multicolumn{3}{|c|}{$\begin{array}{l}\text { Approximate translation between TTree and } \\
\text { RNTuple classes: }\end{array}$} \\
\hline TTree & $\approx$ & $\begin{array}{l}\text { RNTupleReader } \\
\text { RNTupleWriter }\end{array}$ \\
\hline TTreeReader & $\approx$ & RNTupleView \\
\hline TBranch & $\approx$ & RField \\
\hline TBasket & $\approx$ & RPage \\
\hline TTreeCache & $\approx$ & RClusterPool \\
\hline
\end{tabular}

Figure 2. Layers and key class of RNTuple and their approximate counterparts in TTree.

The event iteration layer provides the user-facing interfaces to read and write events, either through RDataFrame [4] or as hand-written event loops. The user interface is presented in more detail in Section 3.

The logical layer splits $\mathrm{C}++$ objects into columns of fundamental types. Its central class is the RField that provides a $\mathrm{C}++$ template specialization for reading and writing of an I/O supported type. Currently there is support for boolean, integer and floating point, std: : vector and std: : array containers, std: : string, std: : variant, and userdefined classes with a ROOT dictionary. In the future, we will provide support for addtional types (e.g., std: : map, std: : chrono) and possibly for intra-event object references as a limited form of pointers. While RNTuple limits I/O support to an explicit subset of $\mathrm{C}++$ types, those types are fully composable (e.g., a user-defined class containing a vector of arrays of another user-defined class).

The primitives layer governs the pool of uncompressed and deserialized pages in memory and the representation of fundamental types on disk. For most fundamental types, the 
Table 1. Sample analyses for performance evaluation. Main differences are the data model (flat or with collections) and the number of required branches (dense or sparse reading).

\begin{tabular}{lll}
\hline LHCb run 1 open data B2HHH & H1 micro dst $[\times 10]$ & CMS nanoAOD June 2019 \\
\hline $18 / 26$ branches $(>75 \%)$ & $16 / 152$ branches $(\sim 10 \%)$ & $6 / 1479$ branches $(<1 \%)$ \\
fully flat data model & event sub collections & event sub collections \\
8.5 million events & 2.8 million events & 1.6 million events \\
$24 \mathrm{k}$ selected events & $75 \mathrm{k}$ selected events & $141 \mathrm{k}$ selected events \\
\hline
\end{tabular}

support for multi-threaded writing (one cluster per thread or task) as well as support for multiple threads reading concurrently from the same range of clusters of an RNTuple. In single-threaded analyses, available idle cores should be used for decompression. We believe that these changes will require very little changes to the user-facing API.

Error handling, for instance in case of device faults or malformed input data, is an important aspect of I/O interfaces. While it is often difficult to recover gracefully from $\mathrm{I} / \mathrm{O}$ errors, the $\mathrm{I} / \mathrm{O}$ layer should reliably detect errors and produce an error report as close as possible to the root cause. To this end, RNTuple throws $\mathrm{C}++$ exceptions for $\mathrm{I} / \mathrm{O}$ errors.

At a later point, we intend to add a limited C API for RNTuple in order to facilitate ROOT data being transferred to 3rd party consumers, such as numpy arrays or machine learning toolkits. To this end, most of RNTuple is implemented not to depend on core ROOT classes, such that a minimal, stand-alone RNTuple I/O library can be built. The functionality of this library will initially be limited to reading simple numerical type fields and vectors thereof.

\section{Performance evaluation}

In this section, we analyze the RNTuple performance in terms of read throughput and file size for typical, single threaded analysis tasks. We use three sample analyses for the benchmarks (see Table 1). Each analysis requires a subset of the available event properties, uses some properties to filter events, and calculates an invariant mass from the selected events. The analyses were implemented using both TTree and RNTuple, each variant optimized for best performance with hand-written event loops [5]. Basket/Page sizes and cluster sizes are comparable between TTree and RNTuple files. The "LHCb" sample is derived from an LHCb Open Data course [6]. The "H1" sample is derived from the ROOT "H1 analysis" tutorial with the original data cloned ten times. The "CMS" sample is derived from the ROOT "dimuon" tutorial using the 2019 nanoAOD format [7] with simulated data. Two dedicated physical nodes, "machine 1" and "machine 2" are used for running the benchmarks (see Table 2). Both machines run CentOS 7 and have ROOT ${ }^{2}$ compiled with gec 7.3. A third dedicated node runs XRootD in version 4.10 and is configured to hold the data on a RAM disk.

\subsection{Storage efficiency}

Figure 4 shows the file format efficiency for the input data of the sample analysis. As expected, the TTree and RNTuple efficiency is very similar on the "LHCb" flat data model. ${ }^{3}$ For "H1" and "CMS", RNTuple shows significantly better efficiency due to the more efficient storage of collections and boolean values. (Approximately half of the difference in file size could be eliminated by using TTree's experimental kGenerate0ffsetMap I/O flag.) Space savings of RNTuple remain even after compression.

\footnotetext{
${ }^{2}$ ROOT branch https://github.com/jblomer/root/tree/ntuple-chep-2019

${ }^{3}$ Note, however, that RNTuple provides substantial improvements in read performance as shown in the next section.
} 
Table 2. Overview of the benchmarking hardware.

\begin{tabular}{lll}
\hline Hardware & Machine 1 & Machine 2 \\
\hline CPU & Xeon Platinum 8260 @ 2.4 GHz & Xeon E5-2630v3 @ 2.4 GHz \\
Memory & DDR4 RDIMM 2933 MHz & DDR4 RDIMM 2133 MHz \\
Optane (NVRAM) & Optane DC 2666 MHz (ext4/DAX) & - \\
SSD (flash) & Intel DC P4510, PCIe 3.1 ×4 & - \\
HDD (spinning) & - & $2 \times$ SAS 7200 RPM (RAID1) \\
Network & - & $1 \mathrm{GbE}$ \\
\hline
\end{tabular}
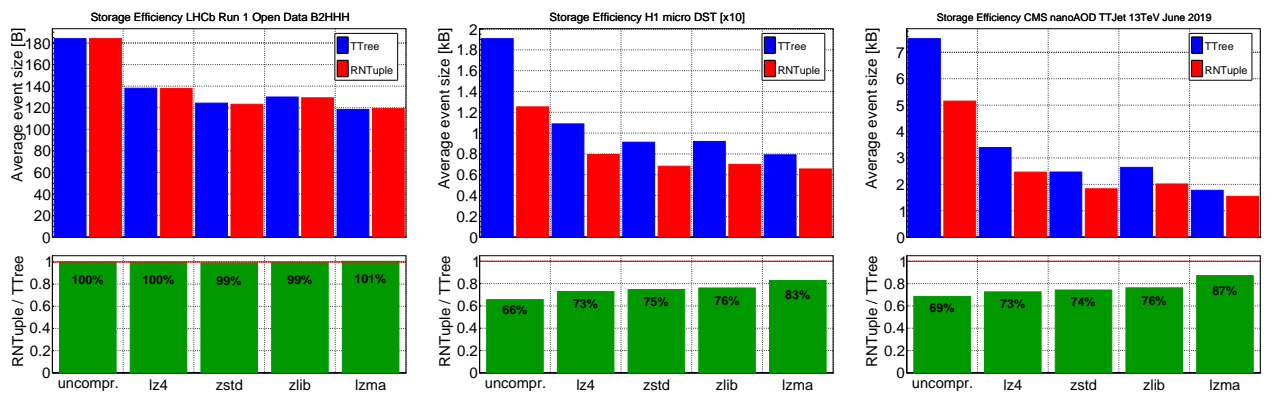

Figure 4. File size comparison of the sample analysis input data in TTree and RNTuple format with different compression algorithms.
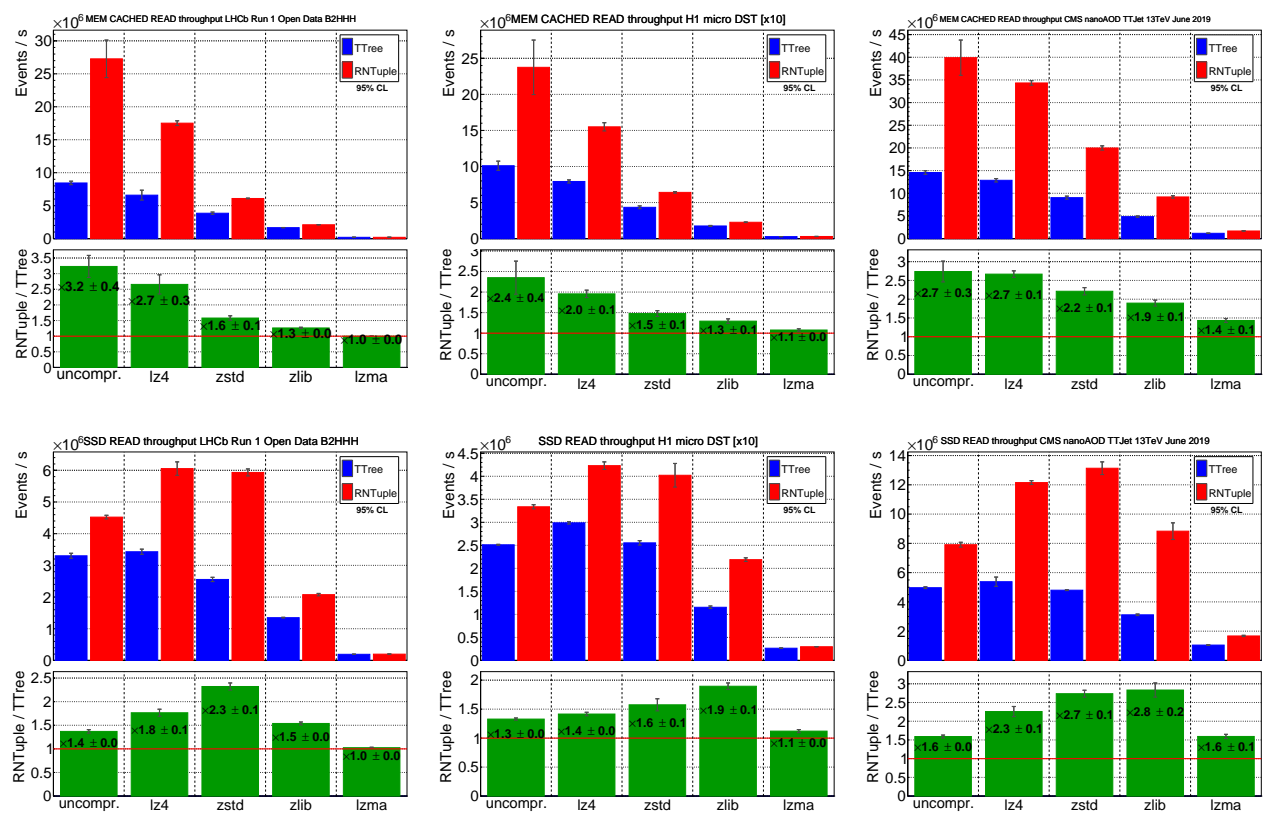

Figure 5. Read throughput in events per second on machine 1. Upper half shows the results for warm file system buffers. Lower half shows the results for reading from SSD with a cold cache. See Figure 7 for further improvements for SSDs. 


\subsection{Read performance}

Figure 5 shows the event throughput for running the sample analyses. When reading from warm file system buffers, the performance is dominated by deserialization and decompression. The data deserialization in RNTuple is significantly faster compared to TTree. With stronger compression algorithms, the performance is more dominated by decompression than by deserialization. Still, even for LZMA compressed data reading RNTuple data is between $10 \%$ and $60 \%$ faster for the $\mathrm{H} 1$ and CMS samples.

When reading with cold file system buffers, as shown in the lower half of Figure 5, the performance depends not only on the deserialization and decompression speed but also on the I/O throughput of the device. The additional CPU time spent on strong compression can be more than compensated by a smaller transfer volume. For RNTuple, there is a sweet spot for the recent zstd compression algorithm, in particular if taking into account the smaller file size as compared to zlib and lz4.

Figure 6 compares cold cache read performance for different, frequently used physical data sources. For the slow devices HDD and $10 \mathrm{GbE}$, the performance is dominated by the I/O scheduler, i. e. by TTreeCache resp. RClusterPool. The I/O scheduler linearizes requests, merges nearby requests, and issues vector reads in order to minimize the overall number of requests sent to a device and the total transfer volume. In these benchmarks the RNTuple's $\mathrm{I} / \mathrm{O}$ scheduler shows a performance at least as good as the TTreeCache.

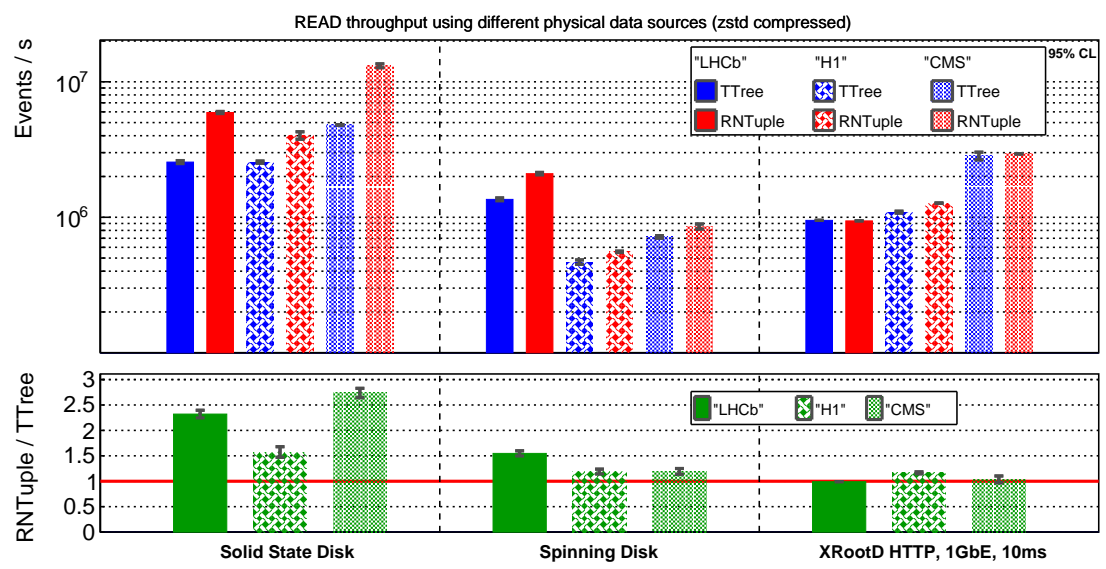

Figure 6. Read speed with different bandwidth and latency profiles. SSD benchmarks from machine 1, HDD and HTTP benchmarks from machine 2 connected to a dedicated, third XRootD server. Note that the SSD results on the left hand side are identical to the SSD/zstd results in Figure 5.

\subsection{SSD optimizations}

In contrast to spinning disks, SSDs are inherently parallel devices that benefit from a large queue depth so that they can read from multiple flash cells concurrently. Figure 7 shows the effect of reading with multiple concurrent streams. To this end, we extend the RNTuple I/O scheduler to read with multiple threads ( 1 stream/thread). Where the read performance is limited by $\mathrm{I} / \mathrm{O}$ and not by decompression and deserialization, increasing the number of streams 


\section{CHEP 2019}

can yield another speed improvement of around a factor of 2.5. The gains max out at around 16 streams. The lower gains for uncompressed LHCb and CMS samples are due to a limitation in the current RNTuple implementation that only preloads a single cluster. It therefore does not provide enough concurrent requests to fill the parallel streams. With implementation of multi-cluster read-ahead, this limitation is going to be removed. An interesting topic of future work is investigating automatic ways of the I/O scheduler to adjust to the underlying physical hardware.

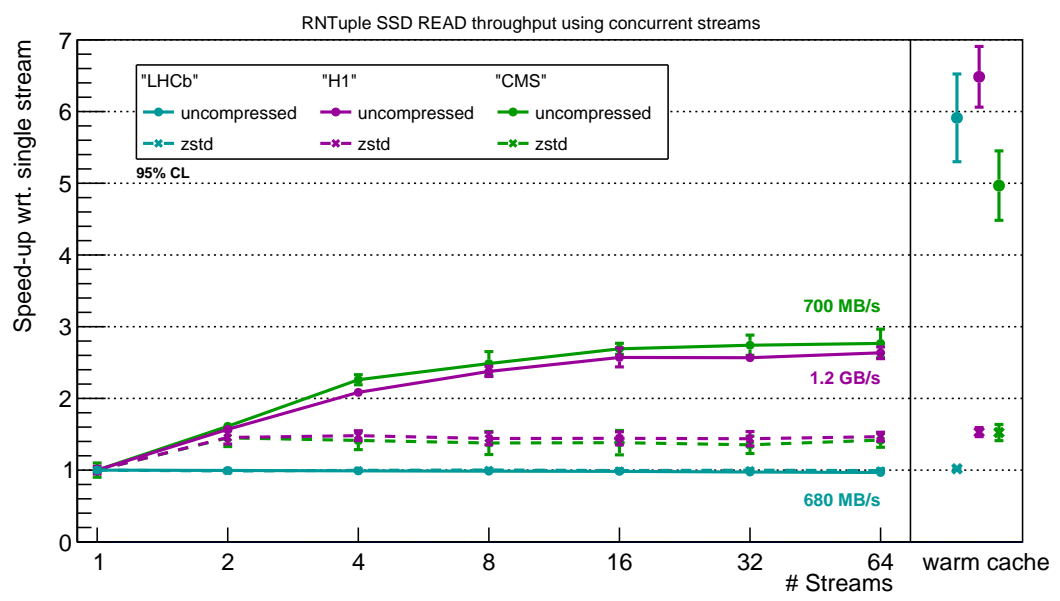

Figure 7. Full exploitation of SSDs by concurrent streams on machine 1. For comparison, the right hand side repeats the warm cache results from Figure 5. Single stream performance is identical to the $\mathrm{SSD} /$ zstd results in Figure 5.

\subsection{Optane DC NV-RAM evaluation}

Figure 8 shows the performance when reading RNTuple data from Optane DC NV-RAMs. The performance characteristics of NV-RAMs are in-between RAM and SSDs (here, we are not exploiting the non-volatility). In the future, they might become a more widespread additional cache layer or installed as a dedicated performance storage tier, e.g. in analysis facilities.

The results show no significant difference between reading from warm file system caches and reading from NV-RAM. As we also do not reach the peak throughput of the NV-RAM modules, the results suggest a bottleneck in the $\mathrm{I} / \mathrm{O}$ deserialization or plotting part of the analysis run. Further optimizations of the RNTuple I/O path are subject of future work.

Due to the fact that the RNTuple on-disk layout matches the in-memory layout, we can compare reading data explicitly with POSIX read() and implicitly by memory mapping. On (byte-addressable) NV-RAM and warm file system buffers, both mechanisms yield comparable results. When reading sparsely from SSDs, the RNTuple I/O scheduler optimizations bring a significant performance gain. Further investigation reveals that the I/O scheduling that underpins the memory mapping in Linux issues an order of magnitude more requests to the device than the RNTuple scheduler. 


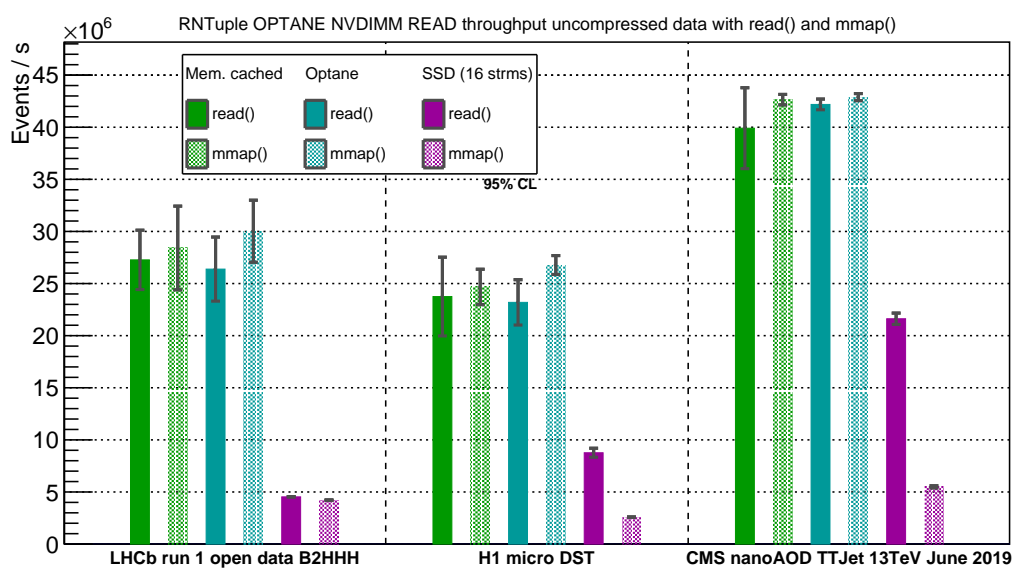

Figure 8. Read performance using Optane DC NV-RAM in "App Direct" mode on machine 1. The NV-RAM block device is formatted with ext4 with DAX optimization. Uncompressed input data is used in order to allow for comparsion between POSIX read() and mmap (). Note that the SSD read() results are identical to the 16 streams result in Figure 7.

\section{Conclusion}

In this contribution we presented the design and a first performance evaluation of RNTuple, ROOT's new experimental event I/O system. The RNTuple I/O system is a backwardsincompatible redesign of TTree, based on the many years of experience of the TTree development. It is from the ground up designed to work well in concurrent environments and to optimally support modern storage hardware and systems, such as SSDs, NV-RAM, and object stores.

Our benchmarks suggest that compared to TTree RNTuple can yield read speed improvements between a factor of 1.5 to 5 in realistic analysis scenarios, while at the same time reducing data sizes by $10 \%$ to $20 \%$. We will gradually move the RNTuple code from a prototype to a ROOT production component. The RNTuple classes are already available in the ROOT : : Experimental : :RNTuple namespace if ROOT is compiled with the root 7 cmake option. Tutorials are available to demonstrate the RNTuple functionality. We consider these developments and the associated future $\mathrm{R} \& \mathrm{D}$ topics essential building blocks for coping with data rates at the HL-LHC.

\section{Acknowledgements}

We would like to thank Fons Rademakers and Luca Atzori from CERN openlab for giving us access to NV-RAM devices. We would like to thank Dirk Düllmann and Michal Simon from CERN IT for providing us an XRootD test node. We would like to thank Oksana Shadura, Brian Bockelman, and Jim Pivarski for many fruitful discussions and suggestions.

\section{References}

[1] R. Brun, F. Rademakers, Nuclear Instruments and Methods in Physics Research Section A: Accelerators, Spectrometers, Detectors and Associated Equipment A 389, 81 (1997) 
[2] J. Blomer, Journal of Physics: Conference Series 1085 (2018)

[3] The Apache Software Foundation, Apache Arrow (2019), https://arrow.apache. org

[4] G. Amadio, J. Blomer, P. Canal, G. Ganis, E. Guiraud, P.M. Vila, L. Moneta, D. Piparo, E. Tejedor, X.V. Pla, Journal of Physics: Conference Series 1085 (2018)

[5] J. Blomer, ROOT RNTuple benchmarks for CHEP 2019 (2019), https : //doi .org/10. $5281 /$ zenodo. 3925770

[6] A. Rogozhnikov, A. Ustyuzhanin, C. Parkes, D. Derkach, M. Litwinski, M. Gersabeck, S. Amerio, S. Dallmeier-Tiessen, T. Head, G. Gilliver (2016), talk at the 22nd Int. Conf. on Computing in High Energy Physics (CHEP'16)

[7] A. Rizzi, G. Petrucciani, M. Peruzzi, EPJ Web Conf 214 (2019) 